\title{
Managing Peritoneal Dialysis Complications through Remote Patient Management Protocols
}

\author{
Ramon Paniagua $^{a} \cdot$ Mario Rojas $^{b} \cdot$ Alfonso Ramos $^{c}$ \\ a Unidad de Investigación Médica en Enfermedades Nefrológicas, Instituto Mexicano del Seguro Social, \\ Ciudad de México, Mexico; ${ }^{\text {b }}$ Departamento de Medicina Interna, Hospital de Especialidades Belisario \\ Domínguez, Secretaría de Salud, Ciudad de México, Mexico; ${ }^{C}$ Medical Affairs, Baxter México, Ciudad de \\ México, Mexico
}

\begin{abstract}
Peritoneal dialysis (PD) has many advantages compared to in-center hemodialysis, which include technical simplicity, and being a home therapy it allows patients significant autonomy for all their daily activities. Nephrologists require trustworthy information from patients for the appropriate management of PD, that is, a careful record of adherence to the prescribed schedule, effective time of dialysis, and a detailed volume of ultrafiltration (UF) is required. All these tasks demand time and commitment from patients, resulting frequently in incomplete or inconsistent information. Development and incorporation of remote monitoring devices to machines for automated PD makes data recovery easy and safe, and provides the medical team the opportunity to be more proactive, and prevent complications due to under dialysis, catheter dysfunction or chronic and acute changes in UF. Remote monitoring devices/machines may be a useful tool in the early diagnosis of peritoneal damage and even in the initial manifestation of peritonitis.

(c) 2019 S. Karger AG, Basel
\end{abstract}

Since its introduction in the late 1970s, peritoneal dialysis (PD) has been recognized as a viable long-term substitutive therapy for renal function [1]. The frequency of use of PD in patients with chronic end-stage renal disease (ESRD) varies widely, from zero in some regions of France and Japan, to $40 \%$ in the United Kingdom, $>60 \%$ in Mexico, and 80\% in Hong Kong [2]. 
In terms of outcomes, PD and hemodialysis (HD) are comparable; mortality in PD is similar and even less than in HD [3, 4]. Nevertheless, the great advantage of PD over $\mathrm{HD}$ is its home application and the technical simplicity, since it allows the patient total autonomy for self-application of the therapy, while also allowing the patient to complete his/her daily productive activities. This advantage is even greater with automatic nocturnal systems or automated PD (APD) [5].

In recent years, the concept of "telemedicine" has been developed, a term which is used to name all electronic transfer of data, audio and video, between health equipment and patients, with the purpose of consulting, examining or performing medical procedures at a distance [6].

Electronic communication facilities have increased the advantages of APD; with the use of telemedicine systems, the rate of hospitalizations has been reduced from 5.7 to 2.2 days, and, in consequence, the costs have also been reduced [7-11]. One worry behind these efforts is to know if patients are prepared to integrate into these systems. Luckily, the results of some surveys indicate that the degree of acceptance is high [12].

Telemedicine systems applied to PD include telephone devices with connection to fixed phones, tablets or teleconferences via the Web. In Japan, telemedicine is used to monitor blood pressure, heart rate, urinary volume, and serum glucose [13], and in Spain, it has been used for teleconferences, for clinical consultation, and in audiovisual presentations for retraining patients [14]. In Canada, contact by tablets has enabled communication between patients and health teams, and through the introduction of alerts in structured interviews, a significant number of hospital visits have been avoided. On the contrary, a high level of patient satisfaction with the system has been achieved [15].

Even when APD is a safe and effective procedure for the treatment of patients with ESRD, the nephrologist depends on an important amount of data which the patient must supply to prepare a prescription adjusted to the clinical conditions of each case. Some aspects are very illustrative, for example: a careful record of adherence to the prescribed schedule, effective time of dialysis, and detailed volumes of ultrafiltration (UF). Monitoring by manual register is liable to the introduction of errors, and the measurements are either incorrect or are incomplete, since it is a tedious job that is less reliable and demands the patient or his/her caregiver's time and additional attention to the connection and disconnection, to register weight and blood pressure or the taking of medications. Development and incorporation of remote monitoring (RM) devices to machines for APD makes data recovery easy and safe, and provides the medical team an opportunity to be more proactive, and prevent complications due to under dialysis, catheter dysfunction or chronic and acute changes in UF [16]. 


\section{RM and PD Adequacy}

Even when it has been established in continuous ambulatory PD patients, that the peritoneal removal of low-weight molecules does not significantly influence patient survival, removal of medium or heavy weight molecules has not been adequately evaluated [17]. It has been established that peritoneal clearance of these molecules is achieved mainly through diffusion, which is associated with the time the dialysis solutions remain in the cavity more than the volume or number of exchanges $[18,19]$. Therefore, patient adherence to the PD prescription is fundamental.

Treatment adherence in patients with chronic diseases is generally very poor, with rates calculated as low as $25 \%$. HD has important advantages that facilitate treatment, as patients have access to more revision and frequent support by nursing staff, more frequent interaction with the nephrologist, and generally more care in the HD center when compared with the patient in PD. In spite of this, non-adherence in HD has a wide range between 22 and 74\%, depending on the time of treatment. For fluid restriction, it has been found in $74 \%$, dietary restrictions $81.4 \%$, and medication prescription up to $73 \%$ [20].

One of the most important advantages of PD is that it is a home treatment that gives the patient the freedom to perform daily activities without depending on a hospital. On the contrary, this independence makes the evaluation of treatment adherence difficult. Usually, this is estimated from the monthly consumption of dialysis solutions and medications, or by self-reporting by the patient. However, both procedures have an important level of subjectivity.

In a systematic review of publications where adherence and non-adherence to treatment in PD patients were studied, wide ranges have been found in both parameters. In regard to dialysis solution exchange, non-adherence varied from 2.6 to $53 \%$, and in medications from 3.9 to $85 \%$, while in diet and fluid restriction, it varied from 14.4 to $67 \%$ [21].

Non-adherence to dialysis treatment is associated with important complications such as an increase in the rate of peritonitis and technique failure, which lead to more hospitalizations and mortality $[22,23]$. New communication techniques offer opportunity for RM and timely detection of voluntary or involuntary digressions in compliance with PD treatment, and allow the implementation of education that reinforces knowledge of the disease by the patient and offers more satisfaction and comfort in following treatment and self-care. Currently, there is little information about the use of RM to evaluate PD treatment adherence.

In a recent study, the frequency of treatment and the difference between the time of dialysis prescribed and real-time treatment was analyzed in 189 patients 
treated for more than 3 months with APD and RM. The results showed that $16.4 \%$ of the patients lost $>10 \%$ of therapy, and $7.4 \%$ of the patients lost more than $20 \%$ of therapy, which means more than 100 and 300 min lost per week [24].

Technology for RM has been available in Mexico for 2 years, which has allowed the detection of the rate of non-adherence to PD prescription. The number of days/week without connection has been monitored in a population of 153 patients with PD without selection by age, gender or cause of ESRD. Simultaneously, they were also questioned by telephone about the number of days without connection in the same period of time. By RM 34.6\% of the patients lost at least 1 day of treatment, $19.0 \%$ lost 2 days of treatment, and $6.6 \%$ more than 2 days of treatment per week. The loss in time per day of dialysis showed 00:56 (hh:mm), 01:06, and more than 03:28, respectively. By telephone interview, the loss of treatment in days per week was $1.0 \pm 1.0$ lower than by RM records [17]. The impact on solute clearances is not available though.

RM of non-adherence to PD prescription allows the detection of inappropriate actions by the patients and the timely implementation of training strategies that reinforce patient knowledge about therapy, thus improving clinical results.

\section{RM and UF}

Maintaining the homeostasis of body fluids is one of the most important goals in dialysis treatment. Fluid overload is the cause of hypertension, abnormal remodeling of the left ventricle, and cardiovascular mortality. It has also been indicated as the cause of edema of the intestinal wall, which facilitates the passage of toxins derived from microbiota through the intestinal wall and the generation of chronic systemic inflammation [25].

In patients on $\mathrm{PD}$, fluid liquid overload is a frequent finding associated with loss of residual renal function and with a positive balance of dialysis solutions in high-transporter patients, among other factors [26]. Fluid overload can be regulated through the proper prescription of glucose concentration in dialysis solutions, or by increasing the number of exchanges and reducing the time of stay in the cavity to maintain the osmotic gradient. The most effective intervention is the use of solutions with icodextrin in the longest exchange.

According to clinical practice guidelines, a minimum volume of $0.75-1.0 \mathrm{~L} /$ day is recommended $[27,28]$, without losing sight of the fact that each $\mathrm{mL}$ of UF associates with absorption of an important amount of glucose, with the consequent metabolic cost. Ideally, the volume of solution infused should be adjusted to the body surface of the patient, taking into account that the total volume in 
the peritoneum may have an additional increase from the UF obtained. The maximum volume in the peritoneum should not exceed the patient's tolerance and should not force the generation of inflammatory stimuli.

\section{Long-Term Variation in UF}

Chronic kidney disease and PD cause important changes in the histology and peritoneal permeability over the long term. It is known that uremia per se induces loss of continuity of the layer of mesothelial cells, thickening of the submesothelial layer, and vascular damage, changes that are more visible in patients with diabetes, patients who also have acute and chronic inflammatory foci. In some reports, it has been noted that these changes, particularly chronic inflammation, are associated with hypoalbuminemia, with higher mortality [29]. PD accentuates the changes proportional to the stay in therapy, probably due to the toxicity derived from exposure to glucose in the solutions.

One of the most important limitations for the chronic use of PD is the increase in peritoneal permeability to small molecules like glucose. The change is presented after many years, and is less important for molecules over $15 \mathrm{kD}$. The clinical manifestation of this phenomenon is the decrease in UF by accelerated diffusion of glucose and loss of osmotic gradient.

\section{Short- and Mid-Term Changes in UF}

In addition to the prolonged exposure to glucose in PD solutions, the reduction in peritoneal UF closely associates with episodes of peritonitis. This relation has been carefully studied since the 1980s. Various reports have shown the increase in creatinine and the reduction in UF in patients that have had peritonitis compared with those who have not suffered this complication. Although the changes in permeability affect small molecules more, the clearance of proteins also decreases, although to a lesser degree [30].

It is important to point out that although the suggested goal of UF has been set at $0.75-1.0 \mathrm{~L} /$ day, the limits for a particular patient may vary according to daily volumes of urine, diet, and the body size of the patient. On the contrary, UF has important variations day to day, although they are small, under the same prescription. The causes for variability have not been clearly identified, but it has been reported that the time the patient remains erect or supine changes the peritoneal surface in contact with the dialysis liquid and locally modifies the concentration gradients and diffusion [31].

RM or telemedicine allows the daily monitoring of UF and the detection of acute or gradual changes over prolonged periods. The means of annual records may allow the evaluation of reduction in UF, commonly attributed to the toxicity of solutions, although there are currently no reports using the new technology. 
Fig. 1. UF values registered by a RM PD device before, during, and after a peritonitis episode in 8 patients. Values from 1 day before and during peritonitis were statistically different from values from 7 days before and after peritonitis $(p<0.05$, by Wilcoxon test). RM, remote monitoring; PD, peritoneal dialysis.

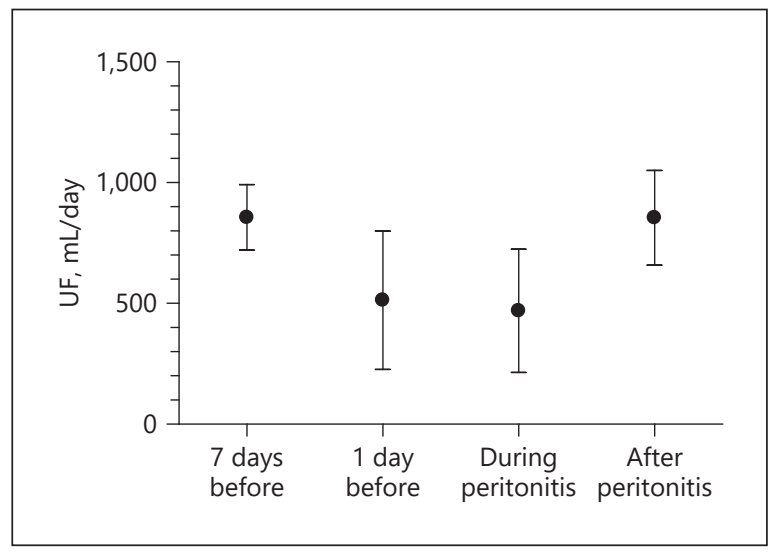

Monthly registers may detect early, temporary or permanent changes derived from frequent complications such as the case of peritonitis, and would allow the necessary adjustments in the prescription to maintain liquid balance [30].

Studies performed in the 1980s show that during episodes of peritonitis, peritoneal permeability increases significantly and returns to baseline values once the infection is cured [32]. Considering that the infection and the onset of immune response precedes by hours or days the appearance of signs and symptoms of peritonitis, it can be hypothesized that UF is immediately reduced before the infection becomes evident.

New RM equipment may be a useful tool for detecting these subtle changes. Before, it should be considered that UF has wide variations from day to day; these have a range of about $10 \%$ of the mean UF. It is also necessary to know the mean UF of each patient to set the limits in the machine for activating alarm signals.

The real potential of RM was analyzed in a retrospective review of 8 patients on device use [33]. Information was collected about the day-to-day UF registered by the device and compared to the mean volumes of the 7 days prior to diagnosis, the day before diagnosis, 2 days after the start of treatment, and 3-7 days after the start of treatment. Figure 1 shows the changes, which were statistically significant between baseline measurements and the days immediately after the start of treatment, with a return to baseline in the 3-7 days after treatment.

Even though these observations are preliminary and limited, they are based on previous knowledge of changes in UF during the evolution of the peritonitis episodes and show that the information collected by RM devices were loaded with individual information of the patient, and with the limits of common variation of daily UF, they may be a useful tool in the early detection of peritonitis. 


\section{References}

1 Popovich RP, Moncrief JW, Nolph KD, Ghods AJ, Twardowski ZJ, Pyle WK: Continuous ambulatory peritoneal dialysis. Ann Intern Med 1978;88: 449-456.

2 USRDS Annual Report 2015. Chapter 13: International Comparisons. Am J Kidney Dis 2016; 67(suppl 1):S291-S334.

3 Mehrotra R, Chiu YW, Kalantar-Zadeh K, Bargman J, Vonesh E: Similar outcomes with hemodialysis and peritoneal dialysis in patients with end-stage renal disease. Arch Intern Med 2011; 171:110-118.

4 Boonpheng B, Thongprayoon C, Cheungpasitporn $\mathrm{W}$ : The comparison of risk of stroke in patients with peritoneal dialysis and hemodialysis: a systematic review and meta-analysis. J Evid Based Med 2018;11:158-168.

5 François K, Bargman JM: Evaluating the benefits of home-based peritoneal dialysis. Int J Nephrol Renovasc Dis 2014;7:447-455.

6 Nayak KS, Ronco C, Karopadi AN, Rosner MH: Telemedicine and remote monitoring: supporting the patient on peritoneal dialysis. Perit Dial Int 2016;36:362-366

7 Gallar P, Vigil A, Rodriguez I, Ortega O, Gutierrez M, Hurtado J, Oliet A, Ortiz M, Mon C, Herrero JC, Lentisco C: Two-year experience with telemedicine in the follow-up of patients in home peritoneal dialysis. J Telemed Telecare 2007;13: 288-292.

8 Nakamoto H, Nishida E, Ryuzaki M, Sone M, Yoshimoto M, Itagaki K: Blood pressure monitoring by cellular telephone in patients on continuous ambulatory peritoneal dialysis. Adv Perit Dial 2004;20:105-110.

9 Edefonti A, Boccola S, Picca M, Paglialonga F, Ardissino G, Marra G, Ghio L, Parisotto MT: Treatment data during pediatric home peritoneal teledialysis. Pediatr Nephrol 2003;18:560-564.

10 Cargill A, Watson AR: Telecare support for patients undergoing chronic peritoneal dialysis. Perit Dial Int 2003;23:91-94.

11 Ghio L, Boccola S, Andronio L, Adami D, Paglialonga F, Ardissino G, Edefonti A: A case study: telemedicine technology and peritoneal dialysis in children. Telemed J E Health 2002;8: 355-359.

12 Lew SQ, Sikka N: Are patients prepared to use telemedicine in home peritoneal dialysis programs? Perit Dial Int 2013;33:714-715.

13 Nakamoto H: Telemedicine system for patients on continuous ambulatory peritoneal dialysis. Perit Dial Int 2007;27(suppl 2):S21-S26.

14 Gallar P, Gutiérrez M, Ortega O, Rodríguez I, Oliet A, Herrero JC, Mon C, Ortiz M, Molina A, Vigil A: [Telemedicine and follow up of peritoneal dialysis patients]. Nefrología 2006;26:365-371.
15 Dey V, Jones A, Spalding EM: Telehealth: acceptability, clinical interventions and quality of life in peritoneal dialysis. SAGE Open Med 2016;4: 2050312116670188.

16 Rosner MH, Lew SQ, Conway P, Ehrlich J, Jarrin R, Patel UD, Rheuban K, Robey RB, Sikka N, Wallace E, Brophy P, Sloand J: Perspectives from the kidney health initiative on advancing technologies to facilitate remote monitoring of patient self-care in RRT. Clin J Am Soc Nephrol 2017;12: 1900-1909.

17 Paniagua R, Amato D, Vonesh E, Correa-Rotter R, Ramos A, Moran J, Mujais S; Mexican Nephrology Collaborative Study Group: Effects of increased peritoneal clearances on mortality rates in peritoneal dialysis: ADEMEX, a prospective, randomized, controlled trial. J Am Soc Nephrol 2002;13:1307-1320.

18 Kabanda A, Goffin E, Bernard A, Lauwerys R, van Ypersele de Strihou C: Factors influencing serum levels and peritoneal clearances of low molecular weight proteins in continuous ambulatory peritoneal dialysis. Kidney Int 1995;48:1946-1952.

19 Steubl D, Roos M, Hettwer S, Angermann S, Wen M, Schmaderer C, Luppa P, Heemann U, Renders $\mathrm{L}$ : Comparison of peritoneal low-molecularweight-protein-removal in CCPD and CAPD patients based on C-terminal agrin fragment clearance. Kidney Blood Press Res 2016;41:175-185.

20 Denhaerynck K, Manhaeve D, Dobbels F, Garzoni D, Nolte C, De Geest S: Prevalence and consequences of nonadherence to hemodialysis regimens. Am J Crit Care 2007;16:222-235.

21 Griva K, Lai AY, Lim HA, Yu Z, Foo MW, Newman SP: Non-adherence in patients on peritoneal dialysis: a systematic review. PLoS One 2014; 9:e89001.

22 Mawar S, Gupta S, Mahajan S: Non-compliance to the continuous ambulatory peritoneal dialysis procedure increases the risk of peritonitis. Int Urol Nephrol 2012;44:1243-1249.

23 Bernardini J, Nagy M, Piraino B: Pattern of noncompliance with dialysis exchanges in peritoneal dialysis patients. Am J Kidney Dis 2000;35:11041110.

24 Firanek C, Salas M, Drummond R. Wiebenson D, Gellens M, Sloand J: Discrepancy between prescribed and actual APD prescription delivery: identification using cycler remote management technology. Nephrol Dial Transplant 2017; 32(suppl 3):iii633.

25 Chen YY, Chen DQ, Chen L, Liu JR, Vaziri ND, Guo Y, Zhao YY: Microbiome-metabolome reveals the contribution of gut-kidney axis on kidney disease. J Transl Med 2019;17:5. 
26 Kim YL, Biesen WV: Fluid overload in peritoneal dialysis patients. Semin Nephrol 2017;37:43-53.

27 Lo WK, Bargman JM, Burkart J, Krediet RT, Pollock C, Kawanishi H, Blake PG; ISPD Adequacy of Peritoneal Dialysis Working Group: Guideline on targets for solute and fluid removal in adult patients on chronic peritoneal dialysis. Perit Dial Int 2006;26:520-522.

28 Woodrow G, Fan SL, Reid C, Denning J, Pyrah AN: Renal association clinical practice guideline on peritoneal dialysis in adults and children. BMC Nephrol 2017;18:333.

29 Contreras-Velázquez JC, Soto V, Jaramillo-Rodríguez Y, Samaniego-Ríos LI, Quiñones-Pérez V, Ávila M, Amato D, Paniagua R: Clinical outcomes and peritoneal histology in patients starting peritoneal dialysis are related to diabetic status and serum albumin levels. Kidney Int Suppl 2008:S34-S41.
30 Yu Z, Lambie M, Davies SJ: Longitudinal study of small solute transport and peritoneal protein clearance in peritoneal dialysis patients. Clin J Am Soc Nephrol 2014;9:326-334.

31 Imholz AL, Koomen GC, Voorn WJ, Struijk DG, Arisz L, Krediet RT: Day-to-day variability of fluid and solute transport in upright and recumbent positions during CAPD. Nephrol Dial Transplant 1998;13:146-153.

32 Krediet RT, Zuyderhoudt FM, Boeschoten EW, Arisz L: Alterations in the peritoneal transport of water and solutes during peritonitis in continuous ambulatory peritoneal dialysis patients. Eur J Clin Invest 1987;17:43-52.

33 Rojas M, Paniagua R, Ramos A: Help of patient remote patient monitoring in the assessment of changes in ultrafiltration before, during and after a peritonitis episode in patients on automated peritoneal dialysis. J Am Soc Nephrol 2016; 27:Abstract Edition, World Kidney Day. Abstract TH-P859. 